\title{
Demixing in hard ellipsoid rod-plate mixtures
}

\author{
Philip J. Camp and Michael P. Allen ${ }^{\text {a) }}$ \\ H. H. Wills Physics Laboratory, Royal Fort, Tyndall Avenue, Bristol, BS8 1TL, United Kingdom
}

Peter G. Bolhuis and Daan Frenkel

FOM Institute for Atomic and Molecular Physics, Kruislaan 407, 1089 SJ Amsterdam, The Netherlands

(Received 10 January 1997; accepted 5 March 1997)

\begin{abstract}
The phase behavior of fluid mixtures of hard uniaxial ellipsoids with elongations $e$ and 1/e, and equal molecular volume, has been studied using constant-pressure Gibbs ensemble Monte Carlo simulations for $e=15$ and $e=20$. Four distinct phases are observed: isotropic (I), uniaxial nematic $\left(\mathrm{N}_{+}\right.$and $\mathrm{N}_{-}$) and biaxial nematic (B). The region of stability of the biaxial phase is found to be limited severely by demixing into two coexisting uniaxial phases. This is in agreement with recent theoretical predictions. The theory, however, does not account for the surprising asymmetry of the phase diagram that we find in our simulations. (c) 1997 American Institute of Physics.
\end{abstract} [S0021-9606(97)50722-7]

\section{INTRODUCTION}

Phase separation in simple binary fluid mixtures is often attributed to van der Waals attractive interactions, because dispersion interactions between like species tend to be stronger than between unlike species. An alternative mechanism for phase separation in binary mixtures, based on nonadditivity of the molecular "hard-core" radii, was put forward by Widom and Rowlinson. ${ }^{1}$ The model system studied by Widom and Rowlinson consisted of two species of spheres (1 and 2) of diameter $\sigma_{11}=\sigma_{22}$. Nonadditivity of hard-core interactions means that $\sigma_{12} \neq\left(\sigma_{11}+\sigma_{22}\right) / 2$. In the WidomRowlinson model $\sigma_{12} \equiv \sigma$ and $\sigma_{11}=\sigma_{22}=0$. In Ref. 1 it was shown that this mixture must undergo a demixing transition at sufficiently high pressures. For a long time, it was generally assumed that a fluid mixture of additive particles would not demix. This belief was based on the work of Lebowitz and Rowlinson, ${ }^{2}$ who showed that, within the PercusYevick closure of the Ornstein-Zernike equation, additive $\left[\sigma_{12}=\left(\sigma_{11}+\sigma_{22}\right) / 2\right]$ hard-sphere mixtures of arbitrary sizeratio are stable with respect to phase separation. However, in the early 90's, experimental data became available that suggested that demixing at high temperatures and pressures might occur, even in systems where the nonadditivity of interactions was believed to be rather small. ${ }^{3}$ Prompted by these findings, Biben and Hansen ${ }^{4}$ re-analysed the problem of phase separation in an additive mixture of hard spheres, using recently developed integral equations for liquid mixtures. This theoretical work provided evidence that fluidfluid phase separation might indeed occur in additive hardcore mixtures. The phase separation is attributed to the osmotic depletion effect, whereby the small spheres are excluded from between two large spheres when the surfacesurface separation is comparable with the small-sphere diameter (for a recent review, see Ref. 5). The osmotic pressure of the small spheres causes an effective attraction between pairs of large spheres. The work of Biben and Hansen inspired new interest in such phenomena and subsequently Frenkel

${ }^{\text {a)} E l e c t r o n i c ~ m a i l: ~ m . p . a l l e n @ b r i s t o l . a c . u k ~}$ and Louis have proved the existence of a first-order demixing transition in a hard-core lattice mixture, ${ }^{6}$ while computer simulation studies have demonstrated phase separation in several additive hard particle fluid mixtures. ${ }^{7,8}$

Binary fluid mixtures of rod-like and plate-like particles exhibit several liquid crystal phases, which are distinguished by the preferred direction of alignment, the director, of each component: the isotropic phase (I); the uniaxial nematic phase $\left(\mathrm{N}_{+}\right)$, occurring in rod-rich mixtures, where the rod director lies along the $z$ axis and the plate director lies in the $x y$ plane; the uniaxial discotic phase $\left(\mathrm{N}_{-}\right)$, occurring in plate-rich mixtures, where the plate director lies along the $z$ axis and the rod director lies in the $x y$ plane; the biaxial phase (B) where the two directors are in a fixed perpendicular relative orientation.

There have been many theoretical studies on such mixtures of hard particles, all of which predict the appearance of a biaxial phase (see e.g. Refs. 9 and 10). Recently, the biaxial phase was observed in computer simulations of hard ellipsoid rod-plate mixtures. ${ }^{11}$ The composition of the mixture is given by the rod fraction $x_{1}=1-x_{2}=N_{1} /\left(N_{1}+N_{2}\right)$, where component 1 refers to the rods and component 2 to the plates. The rods, with elongation $e=10$, and plates, with elongation $e=1 / 10$, were of equal molecular volume, $v_{0}$, so at least the simple "asymmetric" depletion interaction seems unlikely to be present. These simulations were performed with relatively small systems of a few hundred particles, but there was still some evidence of phase separation at the highest densities studied. The measured equations-ofstate and order parameters were compared with a modified Onsager theory, ${ }^{11}$ which showed good agreement. The predicted phase diagram is symmetric about $x_{1}=1 / 2$, since the input of the theory is the second virial coefficient, which is equal for ellipsoids with conjugate elongations and equal molecular volume. ${ }^{12}$ From previous simulation studies of the one-component fluids, there is known to be considerable asymmetry in the phase diagram. ${ }^{13}$

Experimentally, biaxial phases are observed in some micellar solutions (see e.g., Ref. 14) but there is some doubt as 
to whether distinct rod-like and plate-like micelles, or biaxial micelles are formed. Theoretical and simulation studies of rod-plate fluid mixtures ${ }^{10,11,15}$ and biaxial particle fluids ${ }^{16,17}$ have shown the phase diagrams to be isomorphic with one another. In the composition-pressure and biaxiality-pressure phase diagrams of rod-plate fluid mixtures and biaxial particle fluids, respectively, two first-order isotropic-nematic lines meet two second-order nematic-biaxial lines at a bicritical point.

In rod-plate mixtures it has been suggested that the region of stability of the biaxial phase is bounded due to phase separation into coexisting $\mathrm{N}_{+}$and $\mathrm{N}_{-}$phases. ${ }^{18}$ The rodplate mixture is nonadditive, and so phase separation is driven by steric forces. In the context of Onsager theory, van Roij and Mulder ${ }^{19}$ determined the region of stability of the biaxial phase in mixtures of hard rectangular blocks with discretised orientations, conjugate elongations $e$ and $1 / e$, and equal molecular volume, allowing for the possibility of demixing into two uniaxial phases. They found that for $e=5$ the biaxial phase was absent, whereas for $e=15$ there was a small window in the phase diagram where the biaxial phase was stable. The line above which the biaxial phase demixed was found in this study to be parallel with the abscissa of the phase diagram in the $x_{1}-\rho$ plane, where $\rho=\left(N_{1}+N_{2}\right) / V$, the number density. This is purely a result of the truncation of the virial expansion at the second virial coefficient, which in this study was equal for particles with elongations $e$ and $1 / e$, and equal molecular volume. This symmetry is apparent in the phase diagram, since it is symmetric in the $x_{1}-\rho$ plane about the equimolar mixture. It is anticipated that a theory which captures accurately the effect of higher-order interactions would yield an asymmetric phase diagram.

Computer simulations do not rely on any such approximations, and in principle yield essentially exact results. To our knowledge there are no computer simulation data available which address the problem of phase separation in rodplate mixtures. In the present work, we report the results of constant-pressure Gibbs ensemble Monte Carlo (NPT-GEMC) simulations ${ }^{20}$ of hard ellipsoid rod-plate mixtures. For the method to be successful the density must be low enough to ensure sufficient particle transfers between the two simulation cells. As the eccentricity of the particles is increased, the liquid crystal phase transitions occur at lower densities. Realistic molecular elongations fall typically in the range $e=5$ to $e=10$, but to make the NPT-GEMC method tractable we have studied two systems with eccentricities $e=15$ and $e=20$. To aid the interpretation of the simulation results, we calculate the phase diagram using Onsager theory, with Parsons-Lee free energy scaling.

This paper is organised as follows. In Section II we describe the simulations and the calculation of the order parameters used in identifying the phases. The Onsager theory, with the Parsons-Lee free energy scaling, is described briefly in Section III. The results are presented in Section IV and Section $\mathrm{V}$ concludes the paper.

\section{SIMULATIONS}

In this study the prolate ellipsoids are labelled component 1 , and the oblate ellipsoids component 2 . The overall fluid composition is labelled by the rod fraction, $x_{1}=N_{1} / N$. The principal and perpendicular ellipsoid semiaxes of component $i, a_{i}$ and $b_{i}$, were chosen such that $8 a_{i} b_{i}^{2}=1$ and the molecular volume $v_{0}=\pi / 6$. The pressure is reported as $\beta P$, where $\beta=1 / k_{\mathrm{B}} T$. The packing fraction $\eta=\rho v_{0}$, where $\rho$ is the number density, $N / V$.

Constant-pressure Gibbs ensemble simulations ${ }^{20}$ were performed on systems of $N=2000$ ellipsoids with $x_{1}=0.4$ in each box initially: preliminary simulations with $x_{1}=0.5$ failed due to one of the simulation cells emptying. The simulations with $e=15$ were carried out using cubic periodic boundary conditions while those at $e=20$ employed truncated octahedral periodic boundary conditions. In each case the Perram-Wertheim overlap criterion was used. ${ }^{21,22}$ One MC sweep consisted of the following: an attempted translation and rotation of each particle; one attempted volume move in each box; 1000 trial particle transfers, where the particle to be transferred was chosen at random, irrespective of its identity. Any move which resulted in an overlap was rejected and the displacement parameters were adjusted to give a $40-50 \%$ acceptance ratio. The acceptance ratio of the particle transfers was $\mathscr{Q}\left(10^{-5}\right)$ at the highest pressures and densities, which was sufficient given the timescale of fluctuations in the orientational order parameters; long simulations were required for the orientational degrees of freedom to equilibrate. Initial configurations were generated in the isotropic phase and then equilibrated at a series of increasing pressures. For $e=15$ an expansion sequence was also performed, which provided a check for hysteresis. Run lengths were typically $\mathscr{Q}\left(10^{6}\right) \mathrm{MC}$ sweeps per pressure.

NPT-GEMC simulations provide data on the demixed region of the phase diagram, but to examine the transition between isotropic and biaxial phases, or nematic and biaxial phases, single-box constant-pressure simulations were run at constant composition over a range of pressures. Simulations were performed for $e=15$ with $x_{1}=0.52,0.55,0.60,0.63$ and for $e=20$ at $x_{1}=0.5,0.525$. Run lengths were typically Q $\left(10^{6}\right)$ MC sweeps.

\section{A. Order parameters}

The orientational order parameters for this system were calculated within the eigenvector frame of the instantaneous second rank rod order tensor, ${ }^{23,24}$

$$
\mathbf{Q}=\frac{1}{2 N_{1}} \sum_{i=1}^{N_{1}}\left(3 \boldsymbol{\Omega}_{i} \boldsymbol{\Omega}_{i}-\mathbf{I}\right),
$$

where $\boldsymbol{\Omega}$ is the unit orientation vector and $\mathbf{I}$ is the secondrank unit tensor. Order parameters for component $i$ used in identifying isotropic, nematic and biaxial phases are thus defined as follows: ${ }^{10,11}$

$$
\begin{aligned}
& S_{i}=\left\langle P_{2}(\cos \theta)\right\rangle_{i}=\frac{1}{2}\left\langle 3 a_{z}^{2}-1\right\rangle_{i}, \\
& \Delta_{i}=\frac{1}{3}\left\langle P_{2}^{2}(\cos \theta) \cos 2 \phi\right\rangle_{i}=\left\langle a_{x}^{2}\right\rangle_{i}-\left\langle a_{y}^{2}\right\rangle_{i},
\end{aligned}
$$


TABLE I. Characterisation of the isotropic and liquid crystal phases.

\begin{tabular}{ccrrrr}
\hline \hline Phase & Symbol & $S_{1}$ & $\Delta_{1}$ & \multicolumn{1}{c}{$S_{2}$} & $\Delta_{2}$ \\
\hline Isotropic & $\mathrm{I}$ & 0 & 0 & 0 & 0 \\
Uniaxial nematic & $\mathrm{N}_{+}$ & 1 & 0 & $-1 / 2$ & 0 \\
Uniaxial nematic & $\mathrm{N}_{-}$ & $1 / 4$ & $1 / 2$ & $-1 / 2$ & -1 \\
Biaxial & $\mathrm{B}$ & 1 & 0 & $-1 / 2$ & -1 \\
\hline \hline
\end{tabular}

where $a_{\alpha}$ is the projection of the director onto the $\alpha$ axis of the rod eigenvector frame, and $\theta$ and $\phi$ are the corresponding polar and azimuthal angles. Thus the $z$ axis is defined by the rod director. The plates are assumed, by convention, to order along the $y$ axis of this frame. $P_{n}(\cos \theta)$ and $P_{n}^{m}(\cos \theta)$ are, respectively, Legendre and associated Legendre polynomials. In Table I are shown limiting values of the order parameters used in identifying isotropic and liquid crystal phases within the convention adopted here.

In the simulations, the order parameters were calculated by diagonalising the prolate ellipsoid second-rank ordering tensor and evaluating the oblate ellipsoid order parameters within the resultant eigenvector frame, consistent with those outlined above. The rod director, which defines the $z$ axis of the rod eigenvector frame, is identified as the eigenvector corresponding to the largest positive eigenvalue, $\lambda_{+}$. The $y$ axis of the rod eigenvector frame is identified as the eigenvector corresponding to the most negative eigenvalue, $\lambda_{-}$. This choice is arbitrary in the uniaxial phase, where the secondary eigenvectors of the rod orientation tensor are degenerate (apart from finite system size effects). In the biaxial phase, this choice reflects the greater facility for rod orientational fluctuations in the plane perpendicular to the plate director; taking the plates to order along the $y$ axis, the rod eigenvalue corresponding to the $x$ axis will be less negative than that corresponding to the $y$ axis. Thus, the $x$ axis is identified with the middle eigenvalue, $\lambda_{0}$. The prolate ellipsoid order parameter $S_{1}$, corresponds to $\lambda_{+}$while $\Delta_{1}$ corresponds to $\frac{2}{3}\left(\lambda_{0}-\lambda_{-}\right) . S_{2}$ and $\Delta_{2}$ were calculated as projections of the plate orientation vectors onto the rod eigenvector frame. Finite-size effects are expected to be apparent in the order parameters in the sense that, even in the isotropic phase, they will take values of magnitude $\mathscr{Q}(1 / \sqrt{N}) .^{23,25}$

\section{THEORY}

The Onsager theory, with Parsons-Lee free energy scaling, has been described in detail elsewhere ${ }^{11}$ and here we give a brief summary. The Helmholtz free energy of a binary fluid mixture containing $N_{i}=x_{i} N$ particles of component $i$, at reciprocal temperature $\beta=1 / k_{\mathrm{B}} T$ and number density $\rho=N / V$ is

$$
\begin{aligned}
\frac{\beta F}{N}= & \ln \rho \Lambda^{3}-1+x_{1} \ln x_{1}+x_{2} \ln x_{2} \\
& +x_{1} \int \mathrm{d} \boldsymbol{\Omega} \psi_{1}(\boldsymbol{\Omega}) \ln 4 \pi \psi_{1}(\mathbf{\Omega}) \\
& +x_{2} \int \mathrm{d} \mathbf{\Omega} \psi_{2}(\boldsymbol{\Omega}) \ln 4 \pi \psi_{2}(\mathbf{\Omega}) \\
& +\sum_{n=2}^{\infty} \frac{B_{n}}{n-1} \rho^{n-1} .
\end{aligned}
$$

$\psi_{i}(\mathbf{\Omega})$ is the orientational distribution function (ODF) of component $i$, and $B_{n}$ is the $n^{\text {th }}$ virial coefficient. $B_{2}$ is given by

$$
B_{2}=x_{1}^{2} B_{2}^{11}+2 x_{1} x_{2} B_{2}^{12}+x_{2}^{2} B_{2}^{22},
$$

where

$$
B_{2}^{i j}=\frac{1}{2} \int \mathrm{d} \boldsymbol{\Omega} \mathrm{d} \boldsymbol{\Omega}^{\prime} \psi_{i}(\boldsymbol{\Omega}) \psi_{j}\left(\boldsymbol{\Omega}^{\prime}\right) v_{i j}\left(\boldsymbol{\Omega}, \boldsymbol{\Omega}^{\prime}\right),
$$

and $v_{i j}\left(\boldsymbol{\Omega}, \boldsymbol{\Omega}^{\prime}\right)$ is the excluded volume of two particles, one of type $i$ and the other of type $j$, with relative orientations $\boldsymbol{\Omega}$ and $\boldsymbol{\Omega}^{\prime}$. In the original Onsager theory for a pure fluid of nonspherical particles ${ }^{26}$ the summation in Eq. (4) is truncated at $B_{2}$. This approximation becomes exact in the limit $e \rightarrow \infty$, but is inaccurate for intermediate elongations and for $e<1$. To take account of higher order interactions, albeit approximately, we use Parsons-Lee free energy scaling ${ }^{27-29}$ where the Carnahan-Starling excess free energy for hard spheres is scaled by $B_{2} / 4 v_{0}$ :

$$
\begin{aligned}
\frac{\beta F}{N}= & \ln \rho \Lambda^{3}-1 \\
& +x_{1} \int \mathrm{d} \boldsymbol{\Omega} \psi_{1}(\boldsymbol{\Omega}) \ln 4 \pi \psi_{1}(\boldsymbol{\Omega}) \\
& +x_{2} \int \mathrm{d} \boldsymbol{\Omega} \psi_{2}(\boldsymbol{\Omega}) \ln 4 \pi \psi_{2}(\boldsymbol{\Omega}) \\
& +\frac{B_{2}}{4 v_{0}} \frac{\eta(4-3 \eta)}{(1-\eta)^{2}} .
\end{aligned}
$$

In the spherical limit, $e=1, B_{2} / 4 v_{0}=1$ and the CarnahanStarling free energy is recovered, which is known to be accurate to within a few percent of simulation results. ${ }^{30}$ For one-component fluids the I-N transition occurs at lower density as the elongation is increased, and so in the limit $e \rightarrow \infty$ the original Onsager functional is recovered.

The application of this theory to hard ellipsoid rod-plate mixtures is given in Ref. 11. The equilibrium ODF for a density and composition is that which minimises the free energy functional (7), and is found by numerical techniques. ${ }^{11}$ Once the equilibrium ODF is calculated, order parameters and thermodynamic quantities can be calculated and the conditions for phase coexistence solved. ${ }^{11}$ 
TABLE II. Coexistence data from NPT-GEMC simulations with $e=15$ expansion sequence.

\begin{tabular}{cccl}
\hline \hline$\beta P$ & $x_{1}$ & $\eta$ & Phase \\
\hline \multirow{2}{*}{1.62} & 0.386 & 0.164 & $\mathrm{~N}_{-}$ \\
& 0.413 & 0.165 & $\mathrm{~B}$ \\
& 0.374 & 0.175 & $\mathrm{~N}_{-}$ \\
1.80 & 0.424 & 0.174 & $\mathrm{~B}$ \\
& 0.281 & 0.191 & $\mathrm{~N}_{-}$ \\
1.98 & 0.514 & 0.186 & $\mathrm{~B}$ \\
& 0.296 & 0.196 & $\mathrm{~N}_{-}$ \\
2.16 & 0.497 & 0.194 & $\mathrm{~B}$ \\
& 0.179 & 0.220 & $\mathrm{~N}_{-}$ \\
2.34 & 0.624 & 0.209 & $\mathrm{~B}$ \\
& 0.138 & 0.233 & $\mathrm{~N}_{-}$ \\
2.52 & 0.669 & 0.221 & $\mathrm{~B}$ \\
\hline \hline
\end{tabular}

\section{RESULTS}

In Tables II, III, and IV the results of NPT-GEMC simulation are presented, for $e=15$, and $e=20$ respectively. The expansion sequence for $e=15$ is reported in Table II and the compression sequence in Table III. In Figures 1 and 2 we show the phase diagrams in the $x_{1}-\beta P$ plane for $e=15$ and $e=20$, respectively. For $e=15$ at $\beta P>1.62$ the system clearly demixes, while for $e=20$ the demixing occurs for a pressure $\beta P>1.125$. In Figures 3 and 4 we show the phase diagrams in the $x_{1}-\eta$ plane for $e=15$ and $e=20$, respectively. In both cases the demixing transition is clearly indicated. In the $e=20$ case, Figures 2 and 4 also show the $\mathrm{I}-\mathrm{N}_{+}$coexistence data for the pure prolate ellipsoid fluid $\left(x_{1}=1\right)$ determined by thermodynamic integration in Ref. 31. The compression and expansion sequences with $e=15$ show no discernible hysteresis. Equilibration was sluggish, but the absence of any hysteresis in the $e=15$ case indicates
TABLE III. Coexistence data from NPT-GEMC simulations with $e=15$ compression sequence.

\begin{tabular}{|c|c|c|c|}
\hline$\beta P$ & $x_{1}$ & $\eta$ & Phase \\
\hline 1.62 & $\begin{array}{l}0.39(1) \\
0.41(1)\end{array}$ & $\begin{array}{l}0.165(1) \\
0.164(1)\end{array}$ & $\begin{array}{l}\mathrm{N}_{-} \\
\mathrm{B}\end{array}$ \\
\hline 1.80 & $\begin{array}{l}0.36(1) \\
0.43(1)\end{array}$ & $\begin{array}{l}0.178(1) \\
0.177(2)\end{array}$ & $\begin{array}{l}\mathrm{N}_{-} \\
\mathrm{B}\end{array}$ \\
\hline 1.89 & $\begin{array}{l}0.33(1) \\
0.46(1)\end{array}$ & $\begin{array}{l}0.186(1) \\
0.184(1)\end{array}$ & $\begin{array}{l}\mathrm{N}_{-} \\
\mathrm{B}\end{array}$ \\
\hline 1.98 & $\begin{array}{l}0.30(2) \\
0.49(2)\end{array}$ & $\begin{array}{l}0.194(1) \\
0.191(1)\end{array}$ & $\begin{array}{l}\mathrm{N}_{-} \\
\mathrm{B}\end{array}$ \\
\hline 2.05 & $\begin{array}{l}0.26(1) \\
0.528(6)\end{array}$ & $\begin{array}{l}0.201(1) \\
0.196(1)\end{array}$ & $\begin{array}{l}\mathrm{N}_{-} \\
\mathrm{B}\end{array}$ \\
\hline 2.16 & $\begin{array}{l}0.25(2) \\
0.48(3)\end{array}$ & $\begin{array}{l}0.200(7) \\
0.193(7)\end{array}$ & $\begin{array}{l}\mathrm{N}_{-} \\
\mathrm{B}\end{array}$ \\
\hline 2.25 & $\begin{array}{l}0.28(4) \\
0.50(3)\end{array}$ & $\begin{array}{l}0.210(2) \\
0.206(2)\end{array}$ & $\begin{array}{l}\mathrm{N}_{-} \\
\mathrm{B}\end{array}$ \\
\hline 2.34 & $\begin{array}{l}0.21(2) \\
0.56(1)\end{array}$ & $\begin{array}{l}0.216(4) \\
0.208(3)\end{array}$ & $\begin{array}{l}\mathrm{N}_{-} \\
\mathrm{B}\end{array}$ \\
\hline 2.43 & $\begin{array}{l}0.17(4) \\
0.60(2)\end{array}$ & $\begin{array}{l}0.225(3) \\
0.215(3)\end{array}$ & $\begin{array}{l}\mathrm{N}_{-} \\
\mathrm{B}\end{array}$ \\
\hline 2.52 & $\begin{array}{l}0.17(2) \\
0.55(1)\end{array}$ & $\begin{array}{l}0.231(3) \\
0.220(2)\end{array}$ & $\begin{array}{l}\mathrm{N}_{-} \\
\mathrm{B}\end{array}$ \\
\hline 2.61 & $\begin{array}{l}0.123(6) \\
0.569(9)\end{array}$ & $\begin{array}{l}0.238(3) \\
0.224(3)\end{array}$ & $\begin{array}{l}\mathrm{N}_{-} \\
\mathrm{B}\end{array}$ \\
\hline
\end{tabular}

that the simulations were of sufficient length. Also shown in the figures are the results of the Parsons-Lee theory as described in Section III. The theory predicts a phase diagram symmetric about $x_{1}=0.5$, due to the second virial coefficient approximation. By contrast, the simulation data show there to be considerable asymmetry.

TABLE IV. Coexistence data from NPT-GEMC simulations with $e=20$.

\begin{tabular}{|c|c|c|c|c|c|c|c|}
\hline$\beta P$ & $x_{1}$ & $\eta$ & $S_{1}$ & $\Delta_{1}$ & $S_{2}$ & $\Delta_{2}$ & Phase \\
\hline \multirow{2}{*}{1.000} & $0.40(1)$ & $0.116(1)$ & $0.37(8)$ & $0.25(4)$ & $-0.36(2)$ & $-0.62(2)$ & $\mathrm{N}_{-}$ \\
\hline & $0.40(1)$ & $0.116(1)$ & $0.34(5)$ & $0.28(3)$ & $-0.36(2)$ & $-0.65(3)$ & $\mathrm{N}_{-}$ \\
\hline \multirow{2}{*}{1.125} & $0.40(1)$ & $0.128(1)$ & $0.48(5)$ & $0.23(4)$ & $-0.408(7)$ & $-0.71(3)$ & $\mathrm{N}_{-}$ \\
\hline & $0.40(1)$ & $0.128(2)$ & $0.42(3)$ & $0.27(2)$ & $-0.405(8)$ & $-0.73(3)$ & $\mathrm{N}_{-}$ \\
\hline \multirow{2}{*}{1.250} & $0.47(2)$ & $0.137(1)$ & $0.69(4)$ & $0.10(3)$ & $-0.432(6)$ & $-0.61(5)$ & B \\
\hline & $0.32(2)$ & $0.140(1)$ & $0.37(6)$ & $0.34(4)$ & $-0.420(7)$ & $-0.81(2)$ & $\mathrm{N}_{-}$ \\
\hline \multirow{2}{*}{1.375} & $0.49(2)$ & $0.148(1)$ & $0.77(2)$ & $0.06(2)$ & $-0.447(4)$ & $-0.60(8)$ & B \\
\hline & $0.29(3)$ & $0.150(2)$ & $0.41(8)$ & $0.32(5)$ & $-0.434(5)$ & $-0.84(2)$ & $\mathrm{N}_{-}$ \\
\hline \multirow{2}{*}{1.500} & $0.58(4)$ & $0.156(2)$ & $0.85(1)$ & $0.02(1)$ & $-0.457(3)$ & $-0.38(1)$ & $\mathrm{N}_{+} / \mathrm{B}$ \\
\hline & $0.22(2)$ & $0.162(2)$ & $0.36(7)$ & $0.37(5)$ & $-0.446(4)$ & $-0.879(9)$ & $\mathrm{N}_{-}$ \\
\hline \multirow{2}{*}{1.750} & $0.58(2)$ & $0.172(1)$ & $0.87(1)$ & $0.02(1)$ & $-0.465(2)$ & $-0.57(7)$ & $\mathrm{N}_{+} / \mathrm{B}$ \\
\hline & $0.16(1)$ & $0.182(2)$ & $0.36(6)$ & $0.39(4)$ & $-0.459(3)$ & $-0.910(7)$ & $\mathrm{N}_{-}$ \\
\hline \multirow{2}{*}{2.000} & $0.62(3)$ & $0.187(2)$ & $0.903(9)$ & $0.012(5)$ & $-0.471(2)$ & $-0.41(6)$ & $\mathrm{N}_{+} / \mathrm{B}$ \\
\hline & $0.106(8)$ & $0.202(2)$ & $0.36(7)$ & $0.40(4)$ & $-0.467(2)$ & $-0.931(5)$ & $\mathrm{N}_{-}$ \\
\hline
\end{tabular}




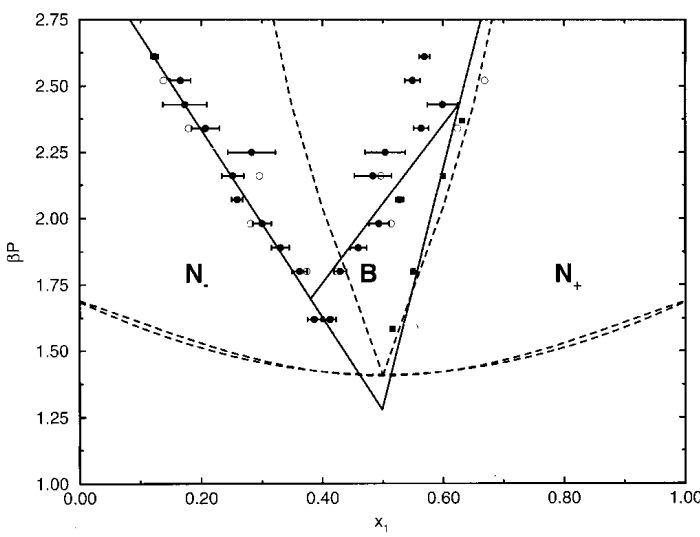

FIG. 1. The phase diagram for $e=15$ in the $x_{1}-\beta P$ plane: Parsons-Lee theory (dashed lines), NPT-GEMC simulations [expansion (open circles), compression (filled circles)], NPT-MC simulations (squares). The solid lines are drawn in to be consistent with the simulation data.

For each pressure simulated, the identities of the two coexisting phases were determined by reference to Table I, and are shown in Tables II, III, and IV. In each case the phase with lower $x_{1}$ is clearly uniaxial discotic $\mathrm{N}_{-}$. The situation in the higher- $x_{1}$ phase is not so clear. On increasing the pressure it is evident that the plate biaxial order parameter, $\Delta_{2}$, decreases, thus signalling a decreasing degree of biaxiality. The values of $\Delta_{2}$ at the highest pressures are considerably lower than those observed in the biaxial phase in Ref. 11. The fact that $\Delta_{2}$ does not fall to zero can be explained in terms of the effect of finite-size on the order parameters. In Section II A it was noted that errors in the order parameters are $\mathscr{Q}(1 / \sqrt{N})$. The reduction of the number of plates in the rod-rich phases will have some effect on the measured values of $\Delta_{2}$. Nonetheless, $\Delta_{2}$ decreases with increasing pressure and therefore at high enough pressures $\mathrm{N}_{-} / \mathrm{N}_{+}$coexistence should occur. The simulation of higher pressures is precluded by the poor acceptance rate of particle transfers.

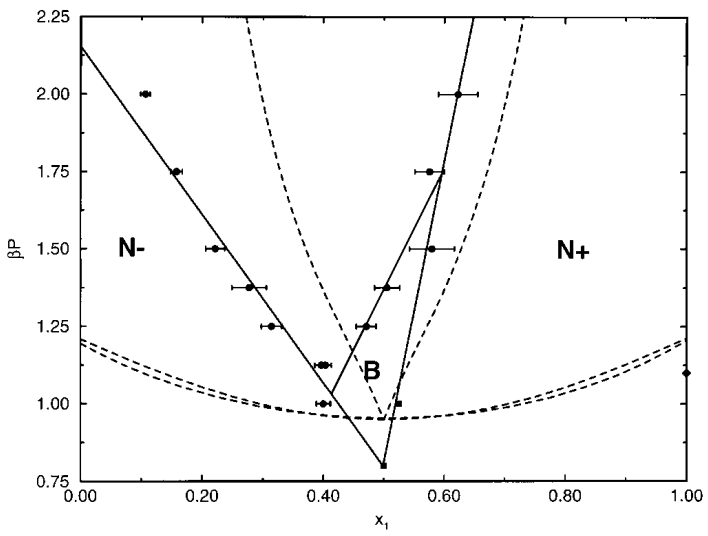

FIG. 2. The phase diagram for $e=20$ in the $x_{1}-\beta P$ plane: Parsons-Lee theory (dashed lines), NPT-GEMC simulations (filled circles), NPT-MC simulations (squares). The diamond at $x_{1}=1$ is from Ref. 31 . The solid lines are drawn in to be consistent with the simulation data.

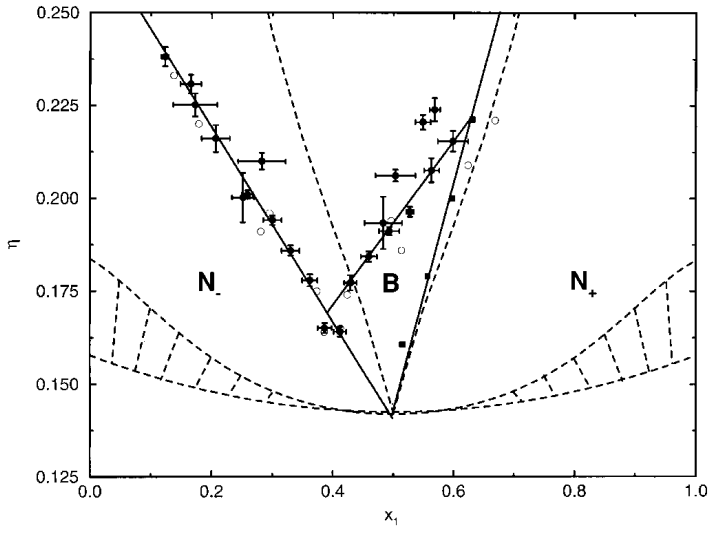

FIG. 3. The phase diagram for $e=15$ in the $x_{1}-\eta$ plane: Parsons-Lee theory (dashed lines), NPT-GEMC simulations [expansion (open circles), compression (filled circles)], NPT-MC simulations (squares). The solid lines are drawn in to be consistent with the simulation data.

The fact that the $\mathrm{N}_{-}$phase can coexist with the $\mathrm{B}$ phase implies that the demixing line is not horizontal, in contrast with the predictions of the Onsager theory. ${ }^{19}$ This is due to the truncation of the virial expansion in the theory, as noted in Section I.

To bracket the region of stability of the biaxial phase, the $\mathrm{N}_{+}-\mathrm{B}$ coexistence line must be located. To this end, single-box constant-pressure MC simulations were perfomed at a range of compositions and pressures, and the order parameters monitored. For a given composition the $\mathrm{N}_{+}-\mathrm{B}$ transition was located on the basis of the biaxial order parameters, $\Delta_{i}$. The results are shown in Figures 1, 2, 3 and 4. In the figures, straight lines have been drawn to indicate the topology of the phase diagram. Clearly, the region of stability of the biaxial phase is small. Onsager theory predicts this region to decrease in size as the elongation of the particles is reduced, ${ }^{19}$ but conventional Gibbs ensemble simulations are intractable at elongations low enough to confirm this.

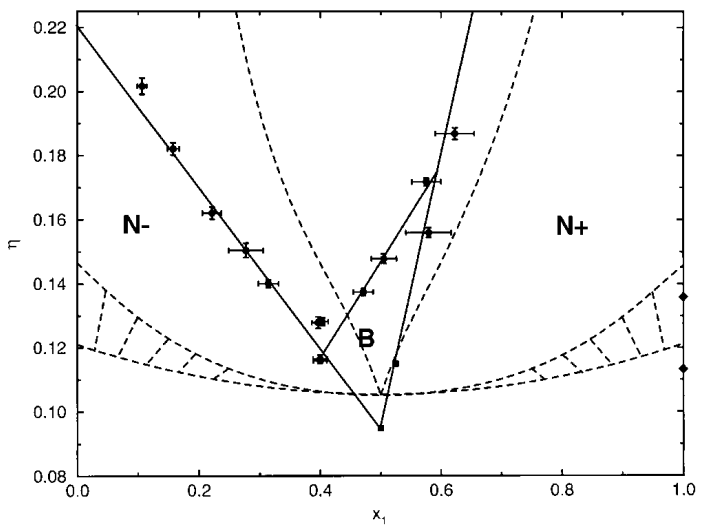

FIG. 4. The phase diagram for $e=20$ in the $x_{1}-\eta$ plane: Parsons-Lee theory (dashed lines), NPT-GEMC simulations (filled circles), NPT-MC simulations (squares). The diamonds at $x_{1}=1$ are from Ref. 31 . The solid lines are drawn in to be consistent with the simulation data. 


\section{CONCLUSIONS}

The range of stability of the biaxial phase with respect to phase separation into two uniaxial nematic phases, in mixtures of hard ellipsoids with conjugate elongation and equal molecular volume, has been investigated by computer simulation. The phase diagrams in the $x_{1}-\beta P$ and $x_{1}-\eta$ planes show the line above which demixing occurs to be skew. A recent Onsager theory predicts this line to be horizontal, but this is a result of the second virial coefficient approximation. The theoretical phase diagrams calculated by van Roij and Mulder, and those calculated here, are symmetric about $x_{1}=1 / 2$ for the same reason. Our simulations confirm that hard-particle mixtures can demix; this is a further counterexample to the hypothesis that attractive interactions are needed to observe such a transition.

Biaxial phases have been observed experimentally in micellar solutions, but it is not clear whether this is due to the presence of micelles with biaxial geometry, or to mixtures of rod-like and plate-like micelles. However, unambiguous rod-plate mixtures could be prepared in colloidal systems. The present work shows that biaxial phases are only expected in a narrow range of compositions and aspect ratios. The results presented here should help the experimentalist in selecting the appropriate conditions to observe stable biaxial phases.

\section{ACKNOWLEDGMENTS}

This research was supported by the Engineering and Physical Sciences Research Council, through grants for the purchase of computer hardware and a research studentship for PJC. The work of the FOM Institute is part of the research program of FOM and is supported by the Nederlandse Organisatie voor Wetenschappelijk Onderzoek (NWO). Discussions between the groups were facilitated by a NATO travel grant.
${ }^{1}$ B. Widom and J. S. Rowlinson, J. Chem. Phys. 52, 1670 (1970).

${ }^{2}$ J. L. Lebowitz and J. S. Rowlinson, J. Chem. Phys. 41, 133 (1964).

${ }^{3}$ A. de Kuijper, B. Smit, J. A. Schouten, and J. P. J. Michels, Europhys. Lett. 13, 679 (1990).

${ }^{4}$ T. Biben and J. P. Hansen, Phys. Rev. Lett. 66, 2215 (1991).

${ }^{5}$ T. Biben, P. Bladon, and D. Frenkel, J. Phys. Condens. Matter 8, 10799 (1996).

${ }^{6}$ D. Frenkel and A. A. Louis, Phys. Rev. Lett. 68, 3363 (1992).

${ }^{7}$ M. Dijkstra and D. Frenkel, Phys. Rev. Lett. 72, 298 (1994).

${ }^{8}$ M. Dijkstra, D. Frenkel, and J. P. Hansen, J. Chem. Phys. 101, 3179 (1994).

${ }^{9}$ R. Alben, J. Chem. Phys. 59, 4299 (1973).

${ }^{10}$ A. Stroobants and H. N. W. Lekkerkerker, J. Phys. Chem. 88, 3669 (1984).

${ }^{11}$ P. J. Camp and M. P. Allen, Physica A 229, 410 (1996).

${ }^{12}$ B. M. Mulder and D. Frenkel, Mol. Phys. 55, 1193 (1985).

${ }^{13}$ A. Samborski, G. T. Evans, C. P. Mason, and M. P. Allen, Mol. Phys. 81, 263 (1994).

${ }^{14}$ L. J. Yu and A. Saupe, Phys. Rev. Lett. 45, 1000 (1980).

${ }^{15}$ R. Hashim, G. Luckhurst, F. Prata, and S. Romano, Liq. Cryst. 15, 283 (1993).

${ }^{16}$ B. M. Mulder, Phys. Rev. A 39, 360 (1989).

${ }^{17}$ M. P. Allen, Liq. Cryst. 8, 499 (1990).

${ }^{18}$ P. Palffy-Muhoray, J. R. D. Bruyn, and D. A. Dunmur, J. Chem. Phys. 82, 5294 (1985).

${ }^{19}$ R. van Roij and B. Mulder, J. Phys. (France) II 4, 1763 (1994).

${ }^{20}$ A. Z. Panagiotopoulos, N. Quirke, M. Stapleton, and D. J. Tildesley, Mol. Phys. 63, 527 (1988).

${ }^{21}$ J. W. Perram, M. S. Wertheim, J. L. Lebowitz, and G. O. Williams, Chem. Phys. Lett. 105, 277 (1984)

${ }^{22}$ J. W. Perram and M. S. Wertheim, J. Comput. Phys. 58, 409 (1985).

${ }^{23}$ R. Eppenga and D. Frenkel, Mol. Phys. 52, 1303 (1984).

${ }^{24}$ C. Zannoni, in The Molecular Physics of Liquid Crystals, edited by G. R. Luckhurst and G. W. Gray (Academic, New York, 1979), Chap. 3, pp. 191-220.

${ }^{25}$ M. P. Allen, G. T. Evans, D. Frenkel, and B. Mulder, Adv. Chem. Phys. 86, 1 (1993).

${ }^{26}$ L. Onsager, Ann. (N.Y.) Acad. Sci. 51, 627 (1949).

${ }^{27}$ J. D. Parsons, Phys. Rev. A 19, 1225 (1979).

${ }^{28}$ S.-D. Lee, J. Chem. Phys. 87, 4972 (1987).

${ }^{29}$ S.-D. Lee, J. Chem. Phys. 89, 7036 (1989).

${ }^{30}$ J.-P. Hansen and I. R. McDonald, Theory of Simple Liquids, 2nd ed. (Academic, London, 1986).

${ }^{31}$ P. J. Camp, C. P. Mason, M. P. Allen, A. A. Khare, and D. A. Kofke, J. Chem. Phys. 105, 2837 (1996). 